\title{
PHOTOELECTRIC POLARIMETRY OF THE TAIL OF COMET IKEY-SEKI (1965 VIII)
}

\section{J. L. Weinberg and D. E. Beeson}

Introduction

With few exceptions, measurements of cometary brightness and polarization have been restricted to regions in or near the coma and therefore to a relatively small range of phase angles. Photoelectric techniques are required for detailed wavelength coverage, whereas large-field photographic techniques are better suited for mapping the large regions of sky spanned by a comet tail. Observations with a small field of view provide high spatial resolution but generally restrict multicolor measurements of brightness and polarization to a small region of the comet. Observations with a large field of view (diameter larger than 1 or 2 deg) provide adequate color and spatial coverage but can result in the loss of detail. A compromise is afforded by Fabry photometry, using a modest telescope of small aperture and relatively large field of view. This method (Fabry 1910, 1943) has been used successfully in photoelectric studies of the light of the night sky (Roach and Pettit 1951; St Amand 1955; Weinberg 1964a; and others) but does not appear to have been used in the study of comets. In this paper we describe post-perihelion measurements of Comet 1965 VIII made on four nights in October-November 1965 using a Fabry photometer atop 3052m Mt. Haleakala, Hawaii, and present detailed results of observations at $5300 \mathrm{~A}$ on October $29,1965$. 
Instrumentation and Observing Procedures

From October 1961 to May 1962 a one-color photoelectric polarimeter was used at Mt. Haleakala to measure the brightness and polarization at $5300 \AA$ of the light of the night sky (zodiacal light, starlight, airglow; Weinberg 1964a). Observations were continued until October 1964 when the instrument was modified for use in a new night-sky observatory established to provide data on the light of the night sky at line and continuum wavelengths from the visjble to the near infrared. Mechanical and electronic changes were made to the polarimeter, including provision for observing at one or more wavelengths. A versatile alt-azimuth mounting and programmable control system made it possible to use automatic, manual, or mixed observing routines, with provision for varying color, scanning rate, directions and position on the sky, and shutter open/closed times. This instrument and facility was used to map brightness and polarization in the tail of Comet 1965 VIII on four nights between October 29 and November 4, 1965.

The photometer utilized a coupled rotating polaroid-half wave synchronous detector to measure the surface brightness (radiance) of the total and polarized components and the plane of polarization* The field of view was defined by a variable-aperture diaphragm in the focal plane of a $14 \mathrm{~cm}$ achromatic doublet. A 2.9 deg diameter field was used for observations of the comet and of the light of the night

\footnotetext{
*Also referred to as orientation of the plane of polarization or
} direction of polarization or azimuth of vibration (Clarke 1974). 
sky during the same period. A field lens was used to focus an image of the objective on the S-II cathode of a DuMont 6291 photomultiplier selected for high signal-to-noise ratio. Wavelength discrimination was provided by sequential observation with narrow-band interference filters (Table 1) which were placed in the partially-collimated beam defined by the equal curvature negative and positive lenses of a Ross zero corrector (St. Amand 1955).

The measured quantities were the total brightness ( $\mathrm{B}_{\text {tot }}$ ), the polarized brightness ( $B_{p o l}$ ), and the plane of polarization $(\chi)$. $B_{\text {pol }}$ was obtained by calibration with a pile-of-plates polarizer (Weinberg 1964b) and a diffuse, unpolarized standard source. This polarizer also permitted a calibration of $\chi$ and made it possible to evaluate instrumental polarization. $B_{\text {tot }}$ and $B_{\text {pol }}$ are equivalent to the Stokes parameters $I\left(=I_{1}+I_{2}\right)$ and $Q\left(=I_{1}-I_{2}\right)$, where $I_{1}$ and $I_{2}$ are orthogonal components of brightness having their electric vectors perpendicular and parallel, respectively, to the scattering plane $\chi$ and $Q$ define the Stokes parameter $U(=Q \tan 2 \chi)$ The degree of polarization, $p$, is that fraction of the total light that is polarized; i.e, $\mathrm{B}_{\text {pol }} / \mathrm{B}_{\text {tot }}$. By convention, $\mathrm{B}_{\mathrm{pol}}$ and $\mathrm{p}$ are negative when the electric vector is parallel to the scattering plane.

Observations of the tail of Comet 1965 VIII were obtained on October 28 and 29 and November 2 and 4, 1965. At other times on these nights, and on other nights during this period, observations of the light of the night sky and of atmospheric extinction were made with the same instrument. All observations were made by 
scanning in azimuth over a region of sky that included the comet as seen (broadened) in the instrument system. Two methods were used to map the tail of the comet:

1. By scanning slowly (generally $0.5 \mathrm{deg} / \mathrm{sec}$ ) back and forth in azimuth at a fixed altitude (elevation). This method was used for multicolor observations; e-g., a clockwise (CW) scan would be made at one color, the return (counterclockwise or CCW) scan would be made at another color, etc until all filters were used, after which the cycle would repeat. In this way, multicolor observations were obtained both along and across the tail of the comet as the comet moved up through the chosen elevation ( 10 or $20 \mathrm{deg}$ ) in the early morning sky.

2. By step-scanning. This method was used for single color or multicolor observations; e.g., a $\mathrm{CW}$ scan would be made at one elevation with one color, the instrument would increase elevation by $I \mathrm{deg}$ and would scan CCW with the same filter or with another filter, etc. until the entire region of sky containing the comet was observed. This method of scanning provided more information across the tail of the comet and made it possible to map the entire tail more frequently.

TABLE 1

Interference Filter Characteristics

Color,
Nominal, $\AA$

*At center of filter, $1.7^{\circ} \mathrm{C}$. 
The Observations

To show the relative positions and brightnesses of the comet and of the main cone of the zodiacal light, we show in Figure 1 the total night sky brightness and plane of polarization obtained on October 30 , 1965 by scanning part of the morning easterly sky in azimuth at $2.5 \mathrm{deg} / \mathrm{sec}$. The main cone of zodiacal light has a maximum brightness near the ecliptic at azimuth $95 \mathrm{deg}$ and Comet 1965 VIII can be seen near azimuth $112 \mathrm{deg}$. In these regions (ecliptic elongation between 32.5 and $33.9 \mathrm{deg}$ ) the plane of polarization varied smoothly with the electric vector having the same direction for the zodiacal light and for the comet; i.e., perpendicular to the scattering plane. The ecliptic was inclined only 2 deg with respect to the vertical when these observations were made. The comet was ideally positioned with respect to the main cone of the zodiacal light, and the separation of the comet from the smoothly varying total brightness is readily accomplished.

Measurements at $5300 \AA$ on October 29.60 UT illustrate one method for delineating detail on the comet. Measurements were made by scanning at $0.5 \mathrm{deg} / \mathrm{sec}$ over a $9 \times 20 \mathrm{deg}$ section of the sky containing the comet: in azimuth, from 105 to $114 \mathrm{deg}(90$ = east), and in elevation, from 0 (horizon)* to $20 \mathrm{deg}$ in steps of $1.0 \mathrm{deg}$. Figure 2 shows the total brightness of background plus comet for elevations 2 to $20 \mathrm{deg}$ as seen at the base of the atmosphere. This

*The Observatory has a depressed horizon of $1.8 \mathrm{deg}$. 


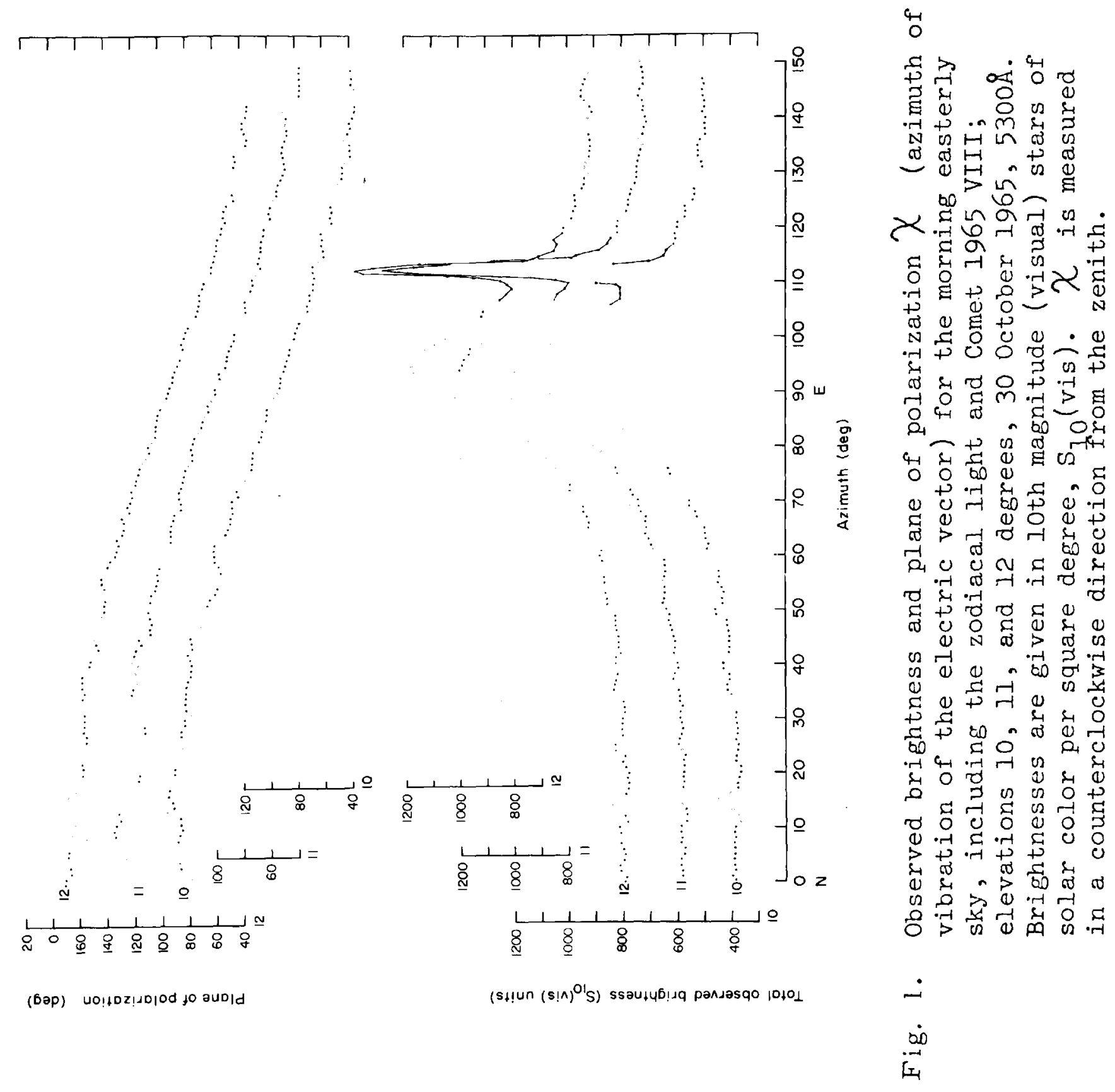







set of observations began at 1432 and ended at 1441 UT on October 29 , with the comet nucleus $5.3 \mathrm{deg}$ below the horizon at the start of the $2 \mathrm{deg}$ elevation scan. A photograph of Comet 1965 VIII taken one day earlier by members of the Haleakala Station of the Smithsonian Astrophysical Observatory was modified by a translation and a rotation to illustrate (Figure 3) the relative positions of the comet and of this set of observations and to compare the photographic appearance of the comet with the way it appeared in the instrument system. The comet aspect is shown for 1435 UT, which corresponds to its position at the end of the $6 \mathrm{deg}$ elevation scan. The comet, horizon (solid line), and scans (even-numbered elevations from 2 through 10) are correct as shown; star positions are not. The width of the comet tail as recorded by the polarimeter is a result of our scanning in azinuth across the tail with a $2: 9$ deg Fabry field of view. The field is uniform and permits resolution of detail smaller than the field size. Figure 4 shows the brightness of the polarized component for the same times and in the same region of sky shown in Figure 2 of particular interest is the change in polarization near $7 \mathrm{deg}$ elevation (approximately $12 \mathrm{deg}$ from the nucleus). Since the background (primarily zodiacal light in this region) and comet radiations are independent, their Stokes parameters are additive. The polarization of zodiacal light throughout this region is "positive"; i.e., the electric vector is perpendicular to the scattering plane. Only negative polarization at distances greater than 12 degrees from the nucleus can produce the observed net decrease in total polarization 


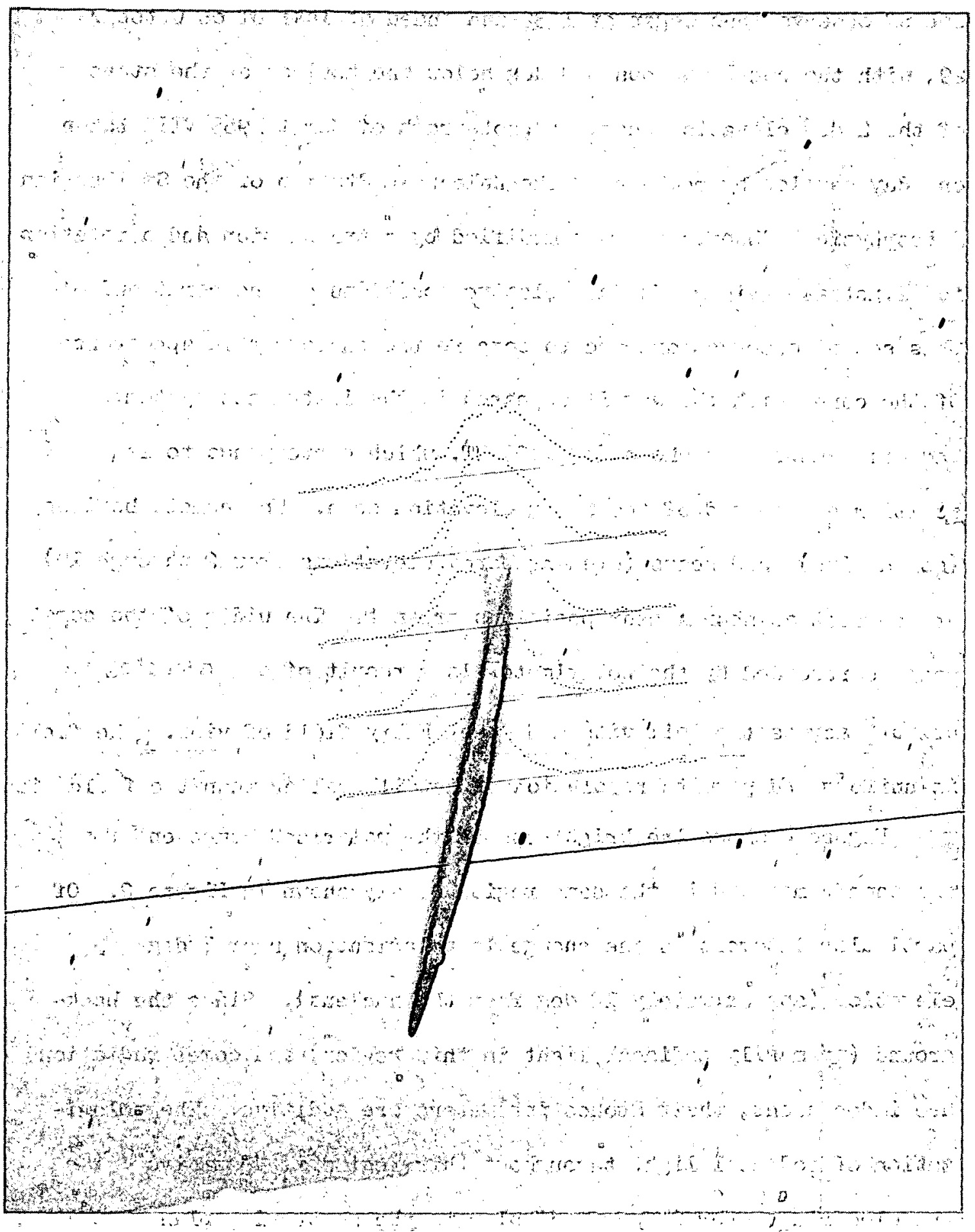

Fig. 3. Comet 1965 VIII aspect at Haleakala together with observed bríghtnesses for éven-numbered elévations from 2 through 10 degrees; 1435 UT on 29 October 1965. 


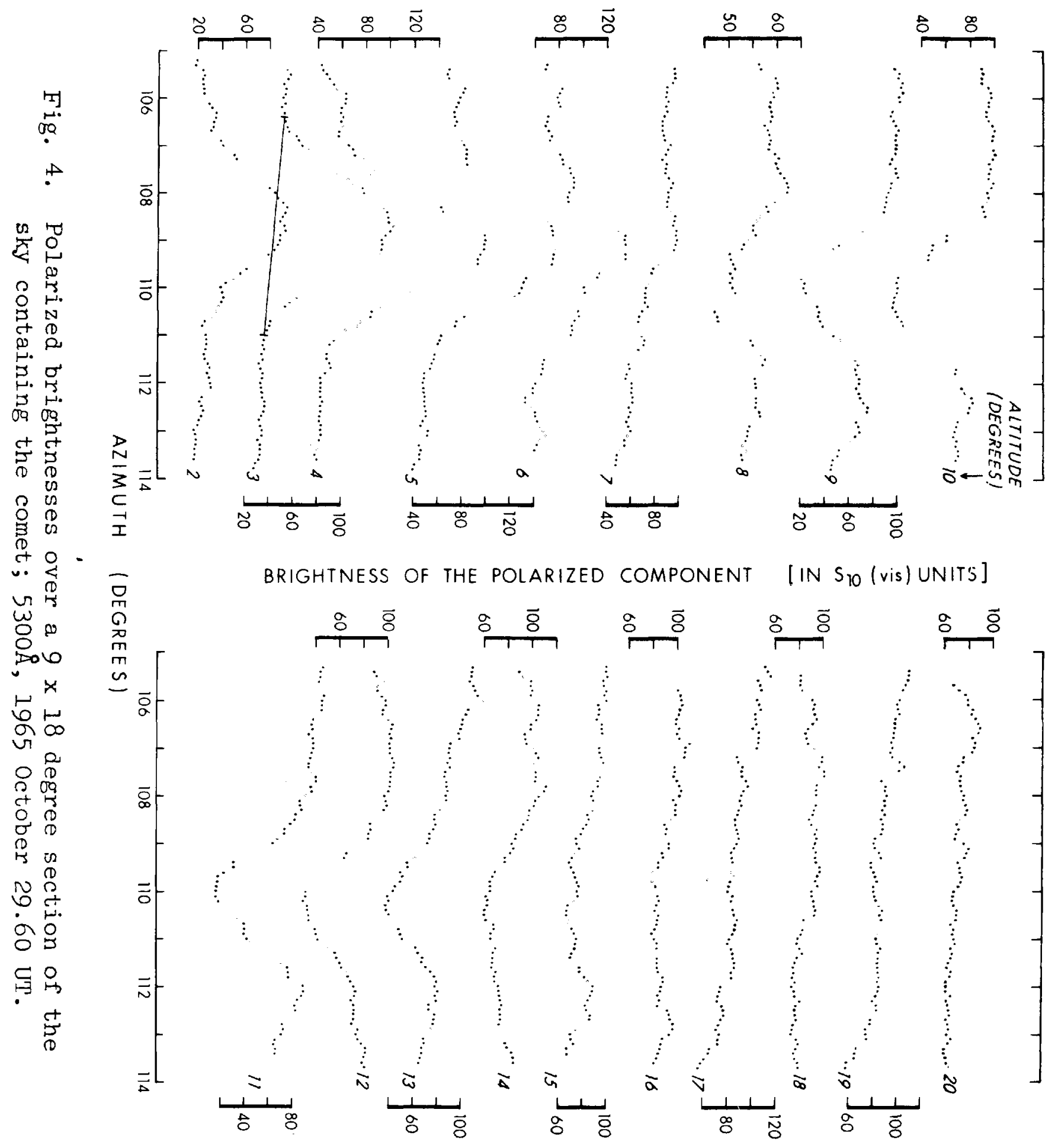


in the direction of the comet tail. This is further illustrated by measurements of the total plane of polarization, $X$ (Figure 5). At elevations 2 through $6 \mathrm{deg}, x$ is the same for the comet and for the zodiacal light: the electric vector is perpendicular to the scattering plane (see, also, Figure 1). At elevations 7 and $8 \mathrm{deg}$ the comet polarization is very small and $X$ is essentially that of the zodiacal light. The fluctuations in $X$ between 9 and 11 deg are a result of the small total polarized brightness in the direction of the tail axis of the comet. There is no reversal in the total polarized brightness, meaning that the polarized brightness of the zodiacal light exceeds the absolute value of the polarized brightness of the comet throughout the region of its negative polarization. The polarized brightness of the comet is still evident at elevation 15 to $16 \mathrm{deg}$, whereas its total brightness is difficult to discern beyond $13 \mathrm{deg}$.

As noted earlier, the separation of the total brightness of the comet from the background is readily accomplished. The irregular variation of the background polarization (Figure 4) makes separation of the polarized brightness of the comet somewhat more difficult. Since the planes of polarization of the comet and of the background were either perpendicular or parallel, it was possible to separate directly the comet polarization $\left(X\right.$ and $\left.\mathrm{B}_{\mathrm{pol}}\right)$ from the total. The separation of the comet's brightness, $B_{\text {tot }}$, from the total does not depend on the planes of polarization. One method used to separate the comet radiation from the background light of the night sky is illustrated by the solid lines shown in Figure 2 (2 deg) and Figure 4 


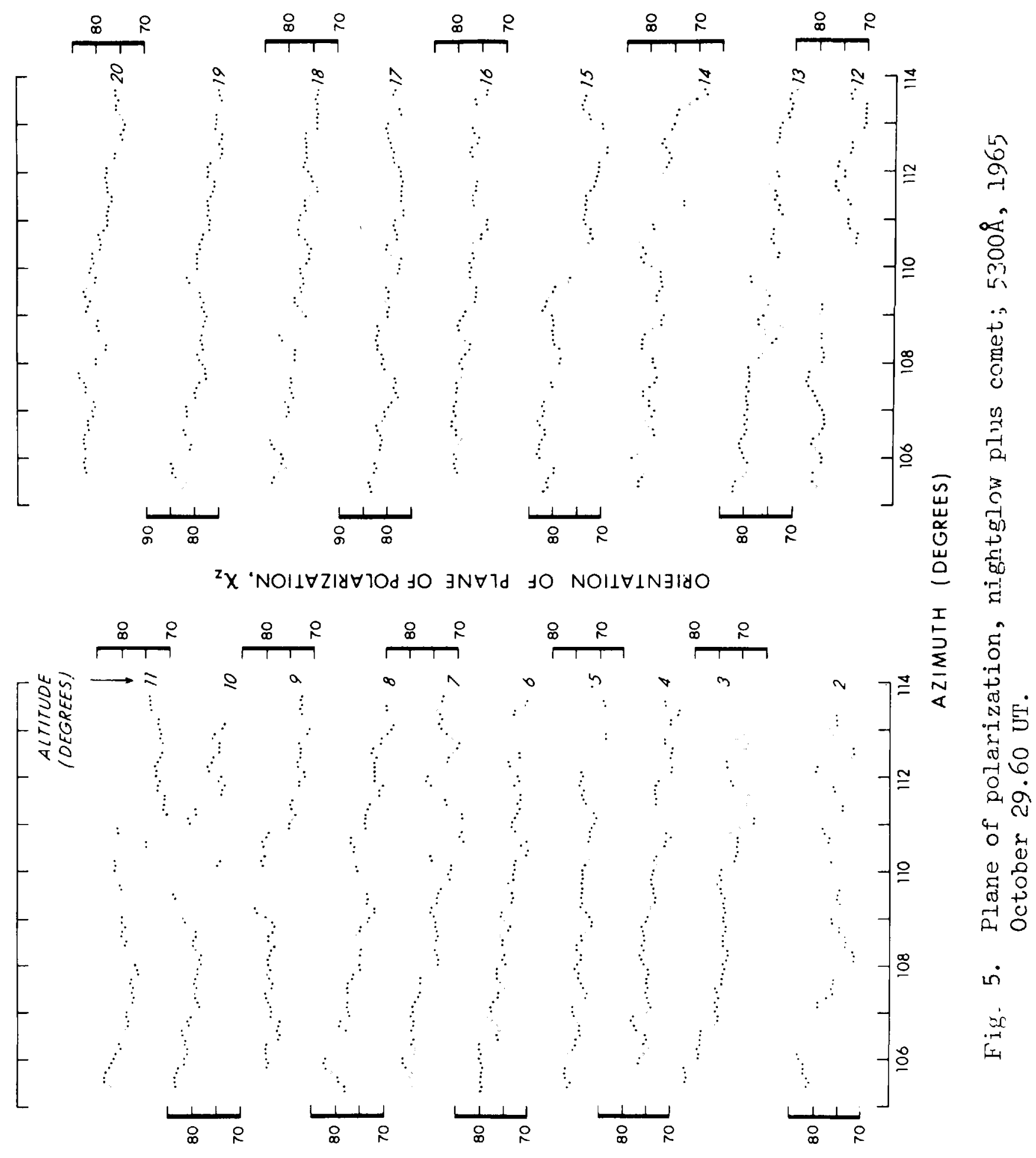


(3 deg) A similar method was used with the raw data recorded at the output of the synchronous detector. The two methods gave equivalent results. Although the total and polarized brightnesses of the comet were visible at elevation 1 deg, they were difficult to separate from the background except near the tail axis. At elevation 0 deg the comet was not visible above the background light of the night sky.

Brightnesses measured in the instrument system were converted to absolute units by reference to a $\mathrm{C}^{1 / 4}$-activated phosphor source calibrated at the Fritz Peak Observatory Photometry Laboratory (Blacker and Gadsden 1967) with an estimated accuracy of $\pm 20 \%$ (Gadsden 1967). Conversion to the equivalent number of 10th magnitude (visual) stars of solar color per square degree, $\mathrm{S}_{10}$ (vis) units*, is based on Johnson's (1954) solar spectral irradiance and on an apparent solar visual magnitude of -26.73 . A further comparison of calibration standards combined with the use of bright stars should improve the accuracy of the calibration to \pm 8 to $10 \%$.

Temperature effects, linearity, and instrument reproducibility were evaluated and the last was checked frequently. A measure of the precision of the observations is given by the dispersion of the measurements in regions where the observations changed only slowly over the sky: standard deviations in $x$ from \pm 0.9 (B $\mathrm{B}_{\text {pol }}$ between 36 and $45 \mathrm{~s}_{10}($ vis $\left.)\right)$ to $\pm 2.0 \mathrm{deg}\left(\mathrm{B}_{\mathrm{pol}}\right.$ between 18 and $\left.28 \mathrm{~s}_{10}(\mathrm{vis})\right)$

\footnotetext{
$* 1 \mathrm{~S}_{10^{(v i s)}}=1.30 \times 10^{-9} \mathrm{ergs} / \mathrm{sec} \mathrm{cm}^{2}$ ster $\mathrm{A}=4.54 \times 10^{-16} \mathrm{~B}$ sun , at $5300 \AA$.
} 
\pm 1.3 to $\pm 1.5 \mathrm{~S}_{10}$ (vis) for $\mathrm{B}_{\mathrm{pol}}$, and $\pm 1.5 \mathrm{~S}_{10}$ (vis) for $\mathrm{B}_{\text {tot }}$. In calibrations and fixed position observations the precision is better. For the levels of total and polarized light measured here by scanning, the precision in degree of polarization, $p$, is typically \pm .01 . For fixed position observations at these same levels, the precision is typically \pm .005 .

Corrections for Atmospheric Extinction and Field of View

Two corrections are required to derive the intrinsic brightness, polarization, and extent of the tail of the comet:

1. Corrections for the diluted brightness observed when the comet does not fill the field of view.

2. Corrections for the effects of atmospheric extinction and scattering.

After the comet brightnesses were separated from the background, they were multiplied by the ratio of the area of the effective (equal area) field of view ( $2.6 \mathrm{deg}$ diameter) to the area of the portion of the tail contained in the field. For instantaneous fields of view centered on the tail axis, this factor was 1.77

In observations of the light of the night sky it is generally assumed that the effects of atmospheric extinction and of light scattered into and out of the fieid of view can be accounted for separately. In this study we neglect atmospheric scattering and treat the comet tail as a point source even though it appears as an extended source when scanned in the instrument system. It is assumed that the atmospheric attenuation can be characterized by the 
product of a coefficient (extinction), ${ }^{\top} \lambda$, that is wavelength dependent and characteristic of the atmosphere as a whole and a path length (air mass), $m_{h}$, which is dependent on elevation $h$. Multiplying the absolute values of total brightness ( $\left.B_{\text {tot }}\right)$ and polarized brightness $\left(B_{\text {pol }}\right)$ by $e^{\top} \lambda m_{h}$ then corrects the values to "outside the atmosphere".

The atmospheric attenuation of bright stars at $5300 \AA$ was measured with the polarimeter on 6 nights between October 25 and November 1; The extinction coefficient, $T$ 5300, derived for the night of October 28/29, 1965 is $.157 \pm .003$ (s.d.) per atmosphere, referred to sea level. Combining the sca level air mass of Bemporad (Bemporad 1907; Schoenberg 1929) with the reduced air mass $(0.690)$ for Mt. Haleakala's height of $3052 \mathrm{~m}$ above sea

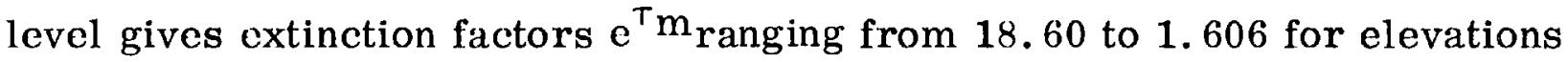
1 to $13 \mathrm{deg}$.

At small elevations it is necessary to take account of the variation of extinction over the field of view. Figure 6 shows instantaneous fields of view centered on the tail axis for elevations 1 and 2 degrees and the large changes in air mass and extinction factor over each field of view. An effective extinction factor was derived by summing the products of $\mathrm{e}^{\top}$ mand area of the comet for $260.1 \mathrm{deg}$ segments contained within the $2.6 \mathrm{deg}$ diameter effective field of view The effective extinction factor was used for elevations $\leq 6 \mathrm{deg}$. For elevations $>6 \mathrm{deg}$, the difference between the center and effective extinction factors is less than 1 per cent and the center value was used. 


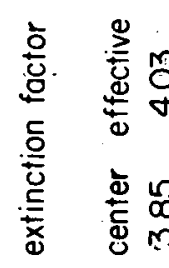

$\begin{array}{lll}\varphi & m & n \\ i & 0 & m\end{array}$

$\begin{array}{lll}\infty & m & 0 \\ \sim & \infty & \infty \\ & \infty & \infty\end{array}$

$\stackrel{\varphi}{\infty} \quad \stackrel{\leftrightarrow}{\stackrel{m}{\infty}}$

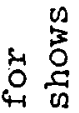

.

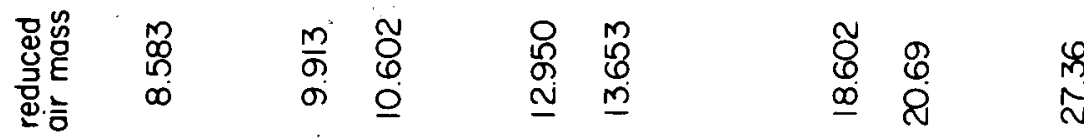

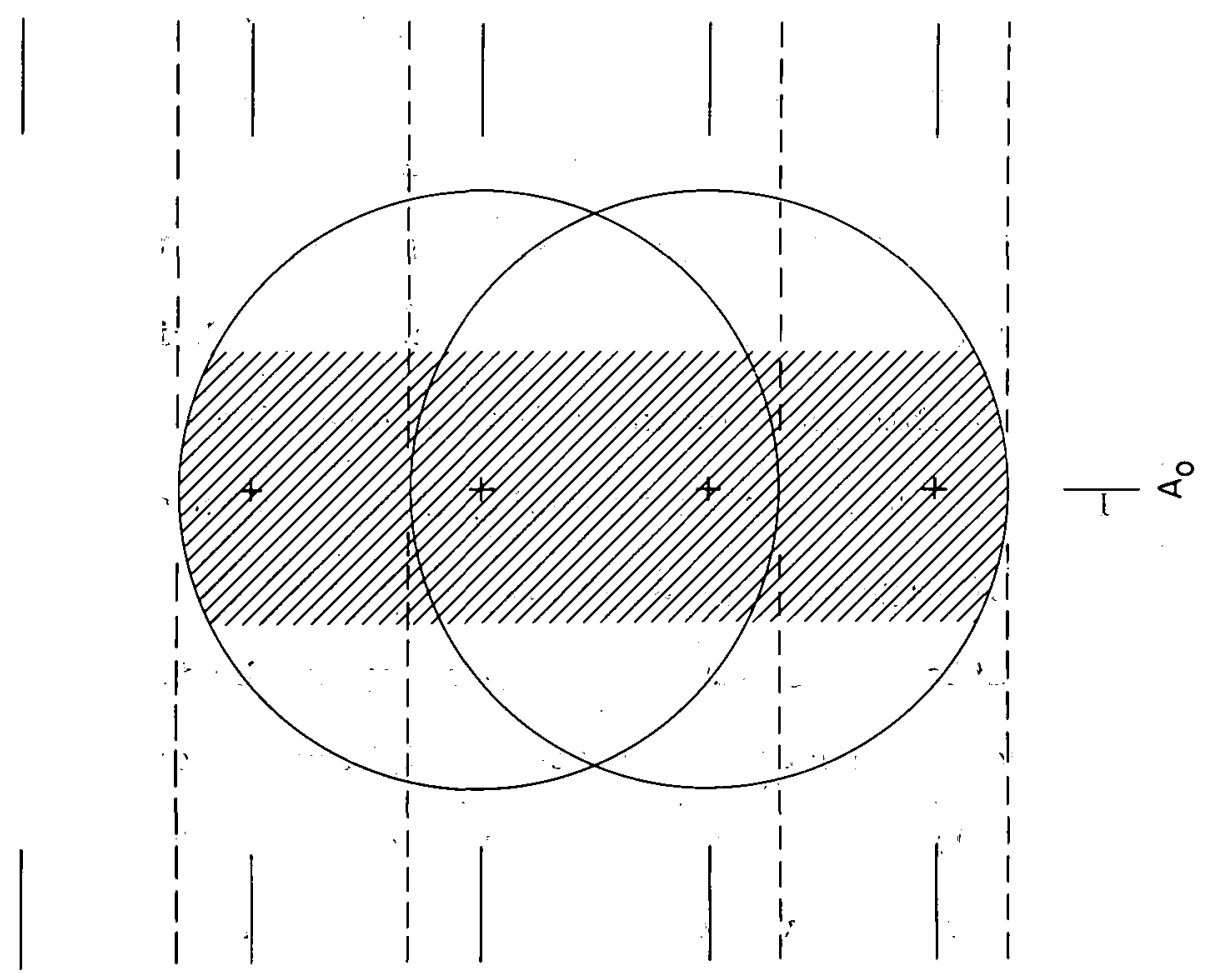



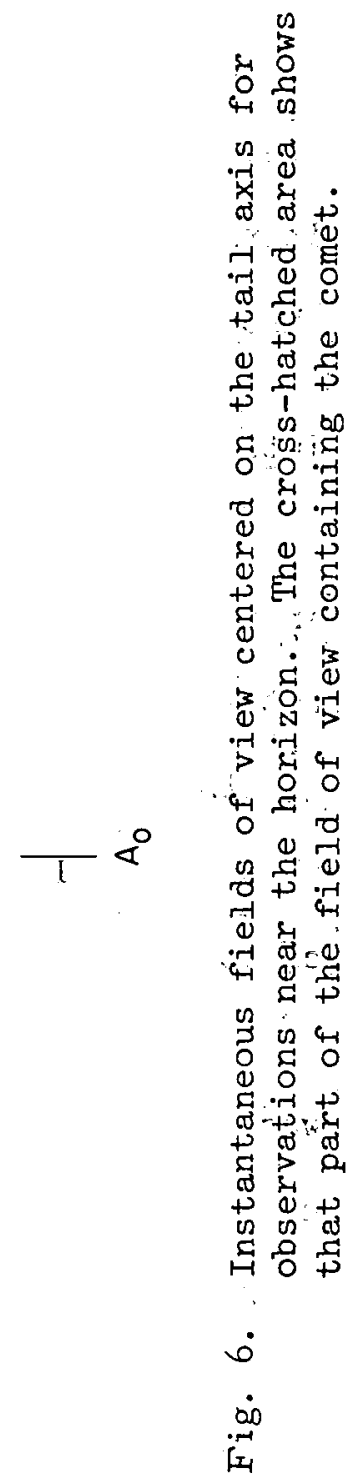


The data cannot be transformed at this point into brightness versus phase angle except along the tail axis where the integrated results are the same in the instrument system and in the comet system. In a subsequent analysis, fields of view off the tail axis will be used in an attempt to derive the variation of brightness and polarization over the field of view (in azimuth and elevation) and, therefore, the intrinsic brightness and polarization of the comet with phase angle throughout the tail.

Integrated Brightness and Polarization of Comet 1965 VIII

A comparison of Figures 2 and 4 shows that the rapid decrease in total brightness with elevation (distance from the nucleus) is not reflected in the rate of decrease of the polarized brightness, and the comet is visible in polarized light several degrees further than in total light. This effect is easily seen even in strip chart recordings of nightglow plus comet.

At each elevation the azimuth corresponding to the tail axis was determined from the shape of the total brightness distribution near the maximum. Phase angles were determined for epoch of date using epherveris elements given by Cunningham (1965) and Roemer (1966) Figure 7 and Table 2 give the integrated brightness and polarization along the tail axis. The error bars in Figure 7 show the maximum effect on $p_{\text {comet }}$ of uncertainties in separating the comet brightnesses ( $\left.B_{\text {pol }}, B_{\text {tot }}\right)$ from the background; the points correspond to the most probable values for the background. The \pm .01 precision in 


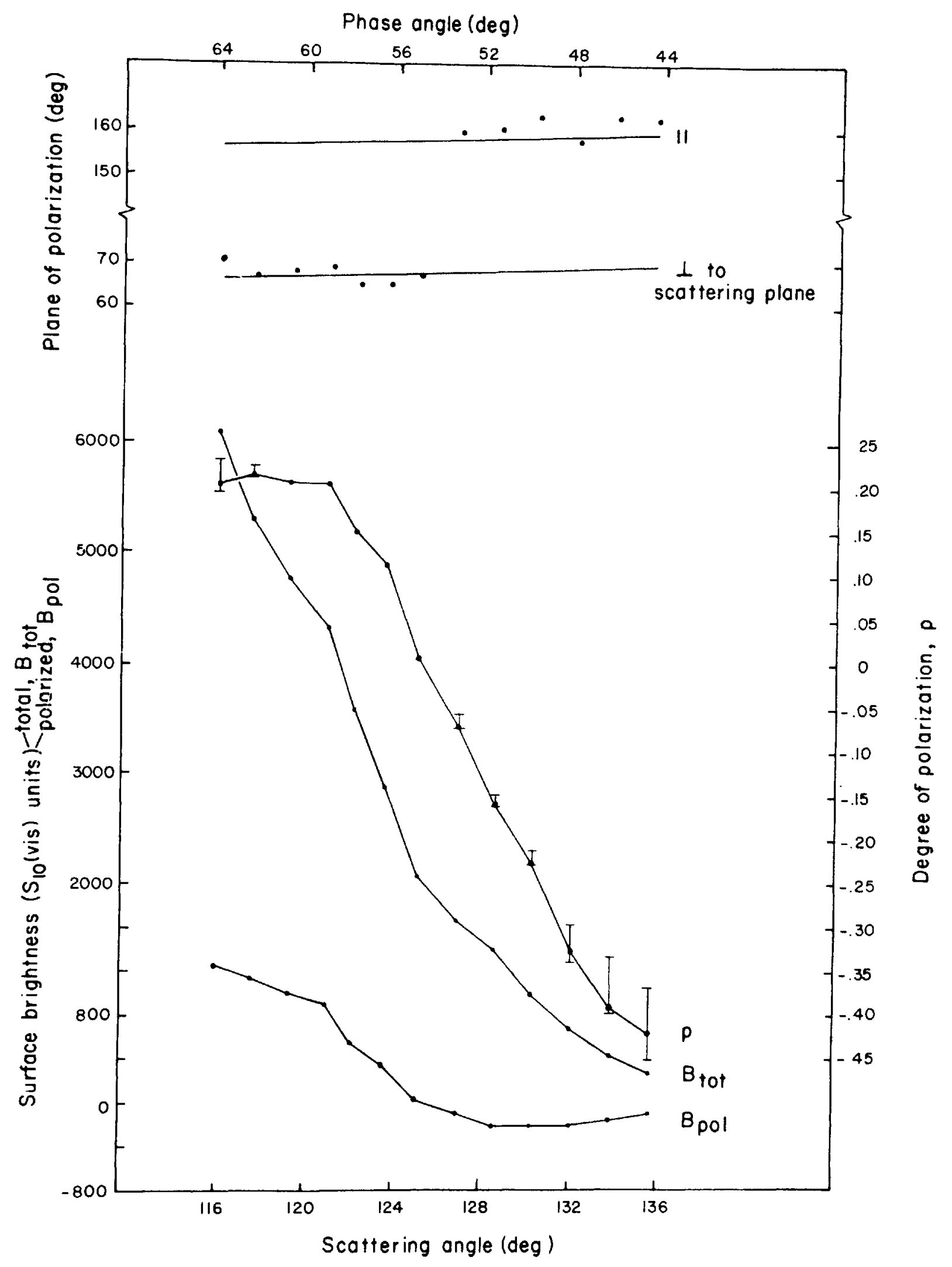

Fig. 7. Integrated brightness and polarization along the tail axis of Comet 1965 VIII; 5300^, 1965 october 29.60 UT. 
TABLE 2

Comet 1965 VIII, 1965 October 29.60 UT; Tail Axis Data, $5300 \AA$

\begin{tabular}{ccccc}
\hline $\begin{array}{c}\text { Elevation } \\
\text { (deg) }\end{array}$ & $\begin{array}{c}\text { Phase } \\
\text { Angle } \\
(\mathrm{deg})\end{array}$ & $\begin{array}{c}\text { Total } \\
\text { Brightnes } \\
\left(\mathrm{S}_{10}(\mathrm{vis})\right)\end{array}$ & $\begin{array}{c}\text { Polarized } \\
\text { Brightness } \\
\left(\mathrm{S}_{10}(\mathrm{vis})\right)\end{array}$ & $\begin{array}{c}\text { Degree of } \\
\text { Polarization }\end{array}$ \\
\hline 1 & 64.0 & 6099 & & \\
2 & 62.4 & 5302 & 1249 & .205 \\
3 & 60.7 & 4762 & 1142 & .215 \\
4 & 59.0 & 4318 & 991 & .208 \\
5 & 57.8 & 3566 & 886 & .205 \\
6 & 56.4 & 2876 & 542 & .151 \\
7 & 54.9 & 2058 & 324 & .113 \\
8 & 53.1 & 1658 & 14 & -.070 \\
9 & 51.4 & 1384 & -116 & -.159 \\
10 & 49.7 & 972 & -221 & -.224 \\
11 & 47.9 & 677 & -217 & -.328 \\
12 & 46.1 & 415 & -221 & -.392 \\
13 & 44.3 & 267 & -163 & -.422 \\
\hline
\end{tabular}

$p$ noted earlier adds .004 to .007 to the errors at elevations 2 through $10 \mathrm{deg}$ and .001 to the errors at other elevations.

The electric vector was found to be perpendicular to the scattering plane for phase angles between 64.0 and 54.9 deg and parallel to the scattering plane for all smaller phase angles. This result contrasts with the photographic observations of Matyagin, et al. (1968) who found the electric vector everywhere perpendicular to the scattering plane

The different rates of decrease of $B_{\text {pol }}$ and of $B_{\text {tot }}$ result in a degree of polarization beyond elevation 2 deg which changes monotonically with distance from the nucleus (with decreasing phase angle). 
At each elevation the degree of polarization shows considerable structure, especially off the tail axis and in regions beyond the neutral point After completing studies relating the comet and instrument systems in off-axis regions, we will examine the appearance of these features at other wavelengths and at $5300 \AA$ at other times and their possible correspondence with the wave-like structures seen in photographs of Comet 1965 VIII.

The different rates of change in the total and polarized brightnesses on either side of elevation $4 \mathrm{deg}$ (phase angle $59.0 \mathrm{deg}$ ) are consistent with the distribution of brightness seen in photographs of Comet 1965 VIII. Other results:

1. In an analysis of all data on the neutral point we found: the neutral point phase angle decreased with time* for each wavelength, the rate of decrease was less at the longer wavelengths, and the phase angle of the neutral point changed from decreasing with wavelength on October 29 to increasing with wavelength by November 2 .

2. The comet was not visible in total or in polarized light with the $5.7 \mathrm{~A}$ filter at $5577 \AA$. A faint enhancement seen with the $10.8 \mathrm{~A}$ filter at $5577 \AA$ can be explained by the increased amount of continuum radiation passed by the broader filter.

3. In a preliminary analysis of morning zodiacal light observations on 8 nights between $5 / 6$ October and $3 / 4$ November, no changes were found that could be attributed to the presence of the newly injected cometary material.

The only other published observations of Comet 1965 VIII far from the nucleus that we are aware of are those of Matyagin, et al. (1968) referred to earlier. The following results were obtained from

* Sekanina (1975) finds that the neutral point appears to follow the motion of the peculiar wave-like structures in the tail 
their photographic observations of polarization using two instruments on 2 November 1965:

1. The polarization was found to be very high, with values of $\mathrm{p}$ far from the nucleus as large as .82 measured with a Meniscus Astrograph and .56 measured with a Schmidt camera.

2. The degree of polarization did not increase monotonically with distance from the nucleus, and it seemed to exhibit

"waves" which were observed with both instruments.

Their data suggest that the wave front was normal to the tail axis. Although structure was observed at each elevation in the Haleakala observations, the values of $\mathrm{p}$ at each elevation were distinctly separated from the values at any other elevation and they increased monotonically with distance from the nucleus.

3. The electric vector was found to be everywhere perpendicular to the scattering plane "to within the errors"

Multicolor observations at Haleakala on 2 November 1965 covered the same regions of the tail observed by Matyagin and his colleagues. Polarization reversal was observed at all colors, including $4355 \AA$ which is close to the approximate effective wavelength of their photographic data.

The authors state that their observations, obtained in the same regions with different instruments and reduced by different methods, "agree well with each other". Yet even a cursory examination of the data tabulated in their paper shows large, systematic differences in the direction and in the degree of polarization obtained in the same regions of the tail with the two instruments and large differences in adjacent observations with the same instrument. 
Matyagin, et al. state that the sky background density was determined by graphical interpolation. If they did not also correct for the sky background polarization, one could explain the large variations in $p$ observed by them in the direction of the comet, especially at large distances from the nucleus, and their failure to detect negative polarization associated with the comet.

\section{Discussion}

The correspondence between the plane of polarization, $X$ comet' and the scattering plane (standard deviation $\pm 2.4 \mathrm{deg}$ ) precludes particle alignment (Harwit and Vanýsek 1971; Clarke 1971) as a significant contributor to the polarization in the tail of comet 1965 VIII.

As noted by a number of authors (Swings 1963; Donn, et al 1967; Vanýsek 1970; and others), multicolor observations of brightness and polarization over a range of scattering angles are required to determine the nature of dust grains in the heads and tails of comets. A review of published model calculations that the authors were aware of and of unpublished results made available by $M$. S. Hanner and by B. Donn shows no model having the features of the observed degree of polarization:

1. The rapid change with phase angle

2. The presence of a neutral point near phase angle $54.8 \mathrm{deg}$.

3. The large range in $p$ (from +.215 to -.422 ).

4. The large amount of negative polarization.

5. The turnover at larger phase angles.

Model calculations of the total and polarized brightnesses of comet tails are few in number; they require as added input the numbers 
of particles and their spatial distribution.

At the scattering angles observed here, Mie scattering may provide information on the properties of the dust grains if they are nearly spherical. Calculations were carried out using a Mie scattering routine developed by Dave (1968) with size distributions of the form

$$
\mathrm{n}(\mathrm{a})=\mathrm{C} \mathrm{a}^{-\mathrm{k}} \quad\left(\mathrm{a}_{\min } \leqslant \mathrm{a} \leqslant \mathrm{a}_{\max }\right)
$$

where $a_{\min }$ and $a_{\max }$ are minimum and maximum radii, respectively. Table 3 lists the input values for refractive index $\mathrm{m}$, size distribution $k$, and particle radii. No assumption was made as to the numbers of particles or their distribution with distance from the nucleus. The routine was used to calculate the total and polarized brightnesses and the degree of polarization over a range of scattering angles for each of the models using an interval of $.01 \mu$ in the integration from $a_{\min }$ to $a_{\max }$.

For almost every model listed under input in Table 3 , the total brightness stayed relatively constant or increased rather than decreased with scattering angle, presumably as a result of our performing the calculations for equal numbers of particles throughout the tail. Although none of the models produced agreement with the observed features of $p$, they did provide information on the effects of each of the parameters on the shape, range, and position of the distribution of $\mathrm{p}$ with scattering angle. For example, only slightly absorbing particles produced the necessary oscillation in $p$ 
TABLE 3

Parameters for Model Calculations, $5300 \AA$

\begin{tabular}{|c|c|c|c|c|}
\hline $\begin{array}{l}\text { Refractive } \\
\text { Index, m }\end{array}$ & $\begin{array}{c}\text { Size } \\
\text { Distribution, }\end{array}$ & $\begin{array}{l}\text { Particle } \\
\qquad \operatorname{Min} \\
(\mu)\end{array}$ & $\begin{array}{l}\text { Radius, a } \\
\qquad \begin{array}{c}\operatorname{Max} \\
(\mu)\end{array}\end{array}$ & $\begin{array}{c}\text { Range of Scattering } \\
\text { Angles, } \theta \\
\text { (deg) }\end{array}$ \\
\hline $\begin{array}{l}\text { input } \\
1.25-.2 i \\
1.25-.2 i \\
1.25-.2 i \\
1.27-1.37 i \\
1.33 \\
1.33-.05 i \\
1.33-.1 i \\
1.33-.2 i \\
1.33-.5 i \\
1.40 \\
1.45 \\
1.45-.05 i \\
1.45-.2 i \\
1.50 \\
1.50 \\
1.50 \\
1.50-.01(.01) .05 i \\
1.50-.03 i \\
1.50-.05 i \\
1.50-.07 i \\
1.50-.07 i \\
1.50-.07 i \\
1.50-.07 i \\
1.50-.1 i \\
1.55 \\
1.55-.05 i \\
1.60 \\
1.65\end{array}$ & $\begin{array}{c}4.0 \\
5.0 \\
2.5(.5) 4 \\
2(.5) 4\end{array}$ & $\begin{array}{c}.1 \\
.1 \\
.1 \\
.05, .1 \\
.05 \\
.1 \\
.1 \\
.1 \\
.1 \\
.05 \\
.05 \\
.1 \\
.1 \\
.05(.02) .11, .15 \\
.2, .4 \\
.6 \\
.05 \\
.11 \\
.07, .09, .15 \\
.1, .15 \\
.05, .1, .15 \\
.4 \\
.4 \\
.4 \\
.1, .2 \\
.05 \\
.1 \\
.05 \\
.05 \\
.05\end{array}$ & $\begin{array}{l}.7, .8 \\
.6(.2) 2 \\
.7, .8 \\
.6(.2) 2 \\
4(.2) 2 \\
.6(.2) 2 \\
.6(.2) 2 \\
.6(.2) 2 \\
.6(.2) 2 \\
.4(.2) 2 \\
.4(.2) 2 \\
.6(.2) 2 \\
.6(.2) 2 \\
.4(.2) 2 \\
.6(.2) 2 \\
1(.4) 3 \\
.4(.2) 2 \\
.4(.2) 2 \\
.4(.2) 2 \\
.6(2) 2 \\
.6(.2) 2 \\
3(1) 6 \\
.6(2) 2 \\
.6(.2) 2 \\
.4(.2) 2 \\
.4(2) 2 \\
.4(.2) 2 \\
.4(.2) 2\end{array}$ & $\begin{array}{l}110(01) 140 \\
90(01) 160\end{array}$ \\
\hline $\begin{array}{l}\text { output } \\
1.255-.200 i \\
1.322-.082 i \\
1.330-.100 i \\
1.397-.064 i \\
1.493-.059 i\end{array}$ & $\begin{array}{r}3.00 \\
-67.48 \\
2.23 \\
-7.15 \\
-9.82\end{array}$ & $\begin{array}{l}.100 \\
.100 \\
.400 \\
.084 \\
.089\end{array}$ & $\begin{array}{l}.802 \\
.600 \\
.802 \\
.600 \\
.551\end{array}$ & \\
\hline
\end{tabular}


(positive to negative to positive, etc in a relatively small range of scattering angle)

An auxiliary routine was written to try to obtain fits to the observed degree of polarization versus scattering angle. Using the aforementioned calculations to suggest starting values for the real and imaginary parts of $\mathrm{m}$, for $\mathrm{k}$, and for $\mathrm{a}_{\min }$ and $\mathrm{a}_{\max }$, a "merit" factor was computed by summing the squares of the differences between the observed and calculated p's at each scattering angle. The value of the first parameter was then changed by a small amount, the merit factor was recalculated, and the derivative of the parameter was taken with respect to the merit factor. The original value of the parameter was incremented by the product of the derivative and the merit factor, and the process was continued until the merit factor decreased. This process was repeated on each of the parameters until the merit factor approached zero.

Figure 8 contains computer plots of the observed degree of polarization (a), one of the input models (b), and several of the calculated p-distributions using the fit program (note, especially, Figures $8 \mathrm{e}$ and $8 f$ ) Although these models may be but a few of the possible combinations that would "fit" the observations, they illustrate several apparent properties of the particles:

1. The range of sizes is very narrow or, equivalently, the size distribution is very steep.

2. The particles have a small imaginary part of their refractive index.

3 The dominant size is of the order of the wavelength of the light 

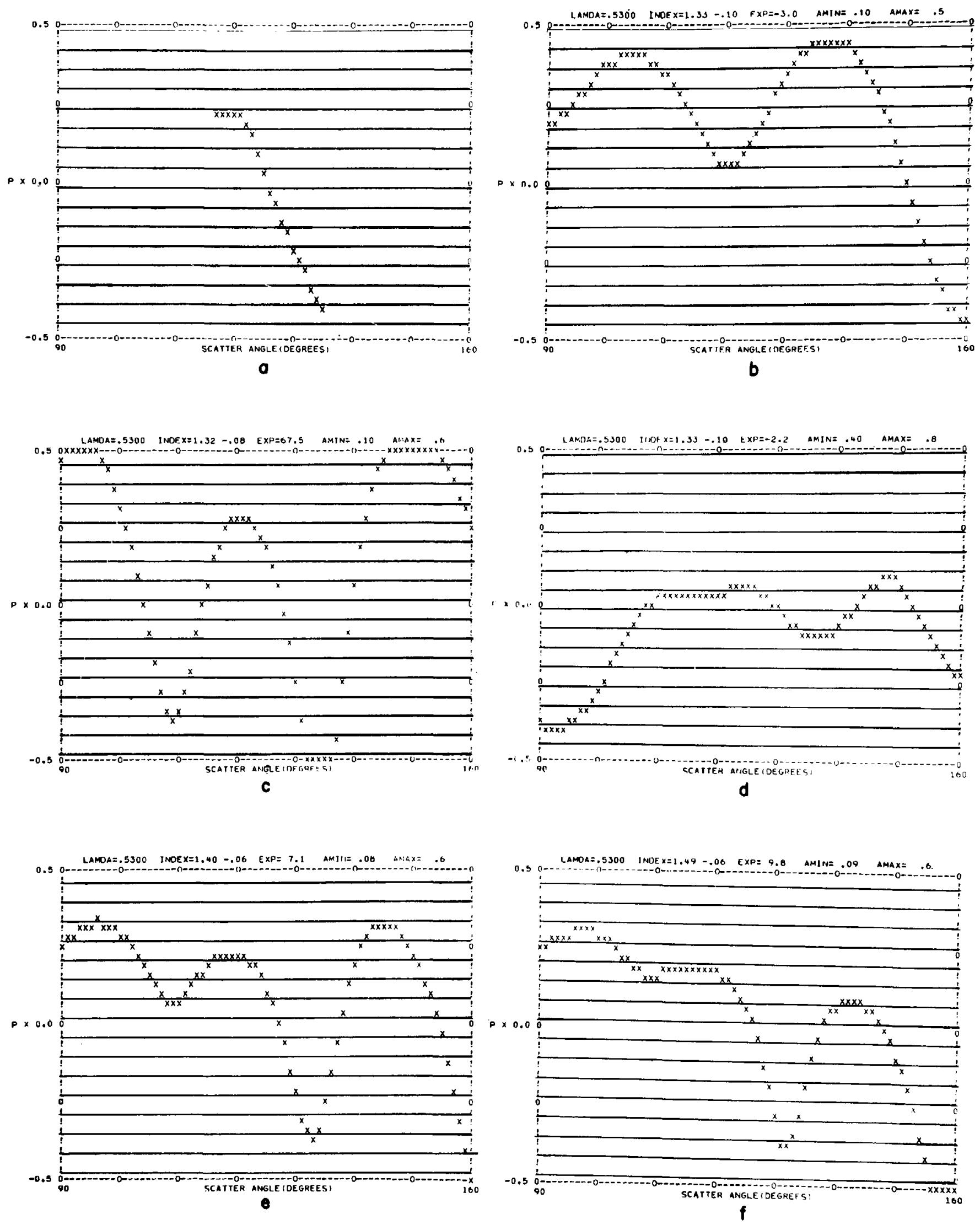

Fig. 8. Computer plots of the observed degree of polarization (a) and of the degree of polarization for selected model calculations (b-f; see, also, Table 3 ). 
A review of our calculations and those of Greenberg (1970), Hanner (unpublished), and Donn and Powell (unpublished) shows further that the decrease that we observe in the phase angle of the neutral point with time would be expected if the particles decreased in size and/or became more absorbing.

This preliminary look suggests that analysis of the remaining data, taken at different times and at different wavelengths, will help to determine the nature of the particles and the short term changes in the averages properties of these particles in the time just after perihelion.

\section{Acknowledgements}

We thank Dr. J. G. Sparrow for detailed discussions of the observations and for his critical reading of the manuscript and Dr J. M. Greenberg for clarifying discussions on the scattering calculations and their use to infer the properties of the grains. Unpublished Mie scattering calculations were kindly made available by $\mathrm{Dr}$ : M. S. Hanner and by Dr. B. Donn. The observations were obtained with the support of NASA grant NsG-676 to the University of Hawaii. The analysis is supported by NASA grant NSG 7093 to State Uniyersity of New York at Albany. 


\section{References}

Bemporad, A. 1907, Versuch einer neuen empirischen Formel zur Darstellung der Anderung der Intensität der Sonnenstrahlung mit der Zenitdistanz, Meteorol. Zeits., 24, 306-313.

Blacker, H. V. and Gadsden, M. 1967, The calibration of airglow photometers, ESSA Technical Memorandum, IERTM-ITSA 85, August

Clarke, D. 1971, Polarization measurements of the head of Comet Bennett (1969i), Astron. and Astrophys., 14, 90-94.

Clarke, D. 1974, Nomenclature of polarized light: linear polarization, Applied Optics, $13,3-5$

Cunningham, L. E. 1965, IAU Circ., No. 1933.

Dave, J. V. 1968, Subroutines for computing the parameters of the electromagnetic radiation scattered by a sphere, Rep. No. 320-3237, IBM Scientific Center, Palo Alto, Calif.

Donn, B., Powell, R. S., and Remy-Battiau, L. 1967, Interpretation of the continuous spectra of comets, Nature, $213,379$.

Fabry: C. 1910, The intrinsic brightness of the starlit sky, Astrophys. J., $31,394-403$.

Fabry, C. 1943, Une méthode de photométrie photographique applicable aux objets ayant un diamètre apparent sensible, Ann. d'Astrophys. 6 , 65-76.

Gadsden, M. 1967, private communication.

Greenberg, J. M. 1970, Models of the zodiacal light, in Space Research X, (Amsterdam: North Holland Publ. Co.), 225-232.

Harwit, M. and Vanýsek, V. 1971, Alignment of dust particles in comet tails, Bull. Astr. Inst. Czech., $22,18-21$.

Johnson, F. S. 1954, The solar constant, J. Meteorol., $\frac{11}{\mathrm{~mm}}, 431-439$.

Matyagin, V. S., Sabitov, Sh. N., and Kharitonov, A. V. 1968, Polarimetry of the tail of Comet Ikeya-Seki, Soviet Astron. -AJ, $\frac{11}{\mathrm{~mm}}$, $863-867$.

Roach, F. E. and Pettit, H. B. 1951, On the diurnal variation of [OI] 5577 in the nightglow, J. Geophys. Res., 56, 325-353. 


\section{References, cont'd}

Roemer, E. 1966, private communication.

Schoenberg, E. 1929, Theoretische Photometrie, in Handbuch der Astrophysik, II/I, $1-280$

Sekanina, Z. 1975, private communication.

St. Amand, P. 1955, Instrumentation for nightglow research, Ann. de Geophys. , 11 , $435-449$

Swings, P. 1963, Scattering by cometary particles, in ICES, Electromagnetic Scattering, (M. Kerker, ed.), 159-169, (New York: Macmillan Co.).

Vanýsek, V. 1970, The behaviour of the polarization of polydisperse interplanetary cloud, Publ. Astr. Inst. Charles University, Prague, No. 58, 3-10.

Weinberg, J. L. 1964a, The zodiacal light at 5300A, Ann. d'Astrophys., $27,718-738$.

Weinberg, J. L. 1964b, On the use of a pile-of-plates polarizer the transmitted component, Applied Optics, $3{ }_{m}^{3}, 1057-1061$. 